\title{
Ultrasonic Doppler Studies of the Vitreous
}

\author{
D. WONG and M. RESTORI \\ London
}

\begin{abstract}
Summary
Simultaneous 'real-time' B-mode and doppler ultrasound techniques were used to study movements of the detached vitreous gel which occurred during duction movements of the eye. The velocity of sound scatterers within the vitreous gel were measured and it was possible to differentiate quantitatively gels which were 'stiff' from those which were 'sloppy'. Information obtained using this technique may throw light on the pathogenesis of rhegmatogenous retinal detachment and assist in the quantitative assessment of retinal mobility in eyes with vitreoretinopathies.
\end{abstract}

'Real-time' B-mode imaging has been used to assess qualitatively the mobility of the detached vitreous gel. ${ }^{1}$ This is particularly impressive in the presence of intragel and/or retrohyaloid opacity. Quantitative assessment of gel collapse using 'real-time' B-mode is, however, tedious and requires a series of still photographs to be assessed at regular time intervals. The B-mode technique uses information concerning both the amplitudes of echoes and the time of arrival of echoes at the transducer but discards information concerning the frequency content of the returned echoes. Doppler techniques however, make use of this information and therefore can be used to determine the velocities of movements of scatterers.

The doppler effect states that ultrasonic pulses will undergo a shift in frequency when reflected or scattered by a moving object. If an idealised case is considered of a single scatterer moving towards the transducer (Fig. 1) then the echoes scattered back to the transducer will be increased in frequency. The doppler shift in frequency $\mathrm{Fd}$, is the difference between the incident and returned frequen- cies and is given by:

$$
\mathrm{Fd}=-2(\mathrm{~V} / \mathrm{C}) \mathrm{Fi} \cos . \theta
$$

and depends on the velocity of movement of the scatterer $\mathrm{V}$, the velocity of the sound within the material surrounding the scatterer $\mathrm{C}$, the frequency of the ultrasonic pulse $\mathrm{Fi}$, and also the angle $\theta$ (Fig. 1) between the pathway of the interrogating pulses and the direction of movement of the scatterer.

Doppler techniques have traditionally been used to study flow within vessels. ${ }^{2}$ Here, an attempt has been made to qualify and quantify the movements of detached vitreous gel using doppler ultrasound.

\section{Material and Methods}

Eyes with detached vitreous gel and with intragel and/or retrohyaloid opacity were selected. The patients were asked to move their eyes quickly to the left and then the right during which simultaneous transverse 'realtime' B-mode studies of the eye and doppler studies of the sound scatterers within the vitreous cavity were made using the Acuson 128 system.* $^{*}$ 
IDEALISED CASE.

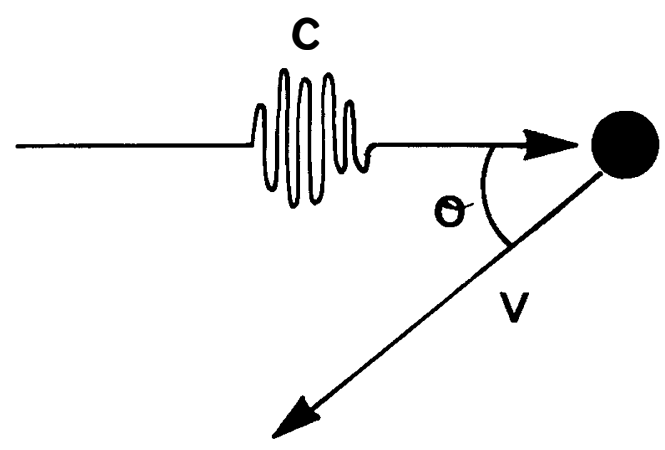

Fig. 1. The Doppler Effect: Ultrasonic pulse of frequency $\mathrm{Fi}$, travelling with velocity $C$, strikes a scatterer travelling with velocity $V$ at an angle $\theta$ to the pathway of the ultrasonic pulse.

\section{'Gate'}

The region of interest for doppler study could be pre-selected on the B-mode image by altering the position and thickness of a window (gate) which is displayed on the B-mode image as two horizontal lines (Fig. 2-top). A wide gate was used to record the velocities of sound scatterers within the whole vitreous gel. A narrow gate was used to study the velocities of movements of scatterers within the anterior, central and posterior parts of the vitreous gel and also of the opacities in the retrohyaloid space.

\section{Output}

The doppler shift in frequency produced by movements of scatterers from within the region pre-selected on the B-mode image, is displayed as a spectrogram. This is essentially a graph of doppler shifted frequencies plotted against time and as the doppler shift in frequency is proportional to the velocity of movement of a scatterer, the graph was calibrated as velocity against time (Fig. 2a). The patients were asked to deviate their eyes quickly to the left and to the right and a range of velocities were recorded. Above the time axis, spots on the spectrogram indicated velocities of scatterers moving towards the transducer, and below the axis moving away from the transducer. As the doppler shifted frequencies were in the audible range, an audible output was also heard.

\section{Results}

\section{Vitreous gel}

Using a wide gate, a typical spectrogram is shown in Fig. 2a. The tracing was biphasic with components above and below the time axis showing velocities towards and away from the transducer respectively. The width of the spectrogram trace indicated the duration for which movements were detected. The components below the axis, that is movements away from the transducer persisted longer. In general, a wide gate encompassing a large portion of the vitreous body produced most information and the spectrogram trace was independent of the direction of movement of the eye. The maximum velocity towards the transducer was recorded as $0.08 \mathrm{~m} / \mathrm{sec}$ and away from the transducer $0.07 \mathrm{~m} / \mathrm{sec}$.

\section{Anterior, central and posterior vitreous gel}

Using a narrow gate, features of the spectrogram from the anterior portions of the vitreous gel was selected. The tracing was biphasic, again with a shorter duration of movements of scatterers towards the transducer than away from it. The total duration of gel motion detected was short (Fig. 2b). Moving a narrow gate more centrally within the gel produced a trace which was dependant upon the direction of gaze and movement and was either entirely towards, or entirely away from the transducer (Fig. 2c). With a narrow gate more posteriorly placed within the gel the spectrogram reverted to a biphasic trace.

\section{'Sloppy' gel}

Mobile or 'sloppy' gels measured with a wide gate produced spectrograms as shown in Figures $2 a$ and $3 a$. The scatterers within the gel move with varying velocities in different directions to produce a complicated spectrogram.

\section{'Stiff' gel}

Using a wide gate, the spectrogram from a relatively immobile ('stiff') gel is shown in Figure $3 b$. The tracing is simple; symmetrical in shape and short in duration. This was a reflection of the corporate mobility of the scatterers confined within the firm gel. The gel 

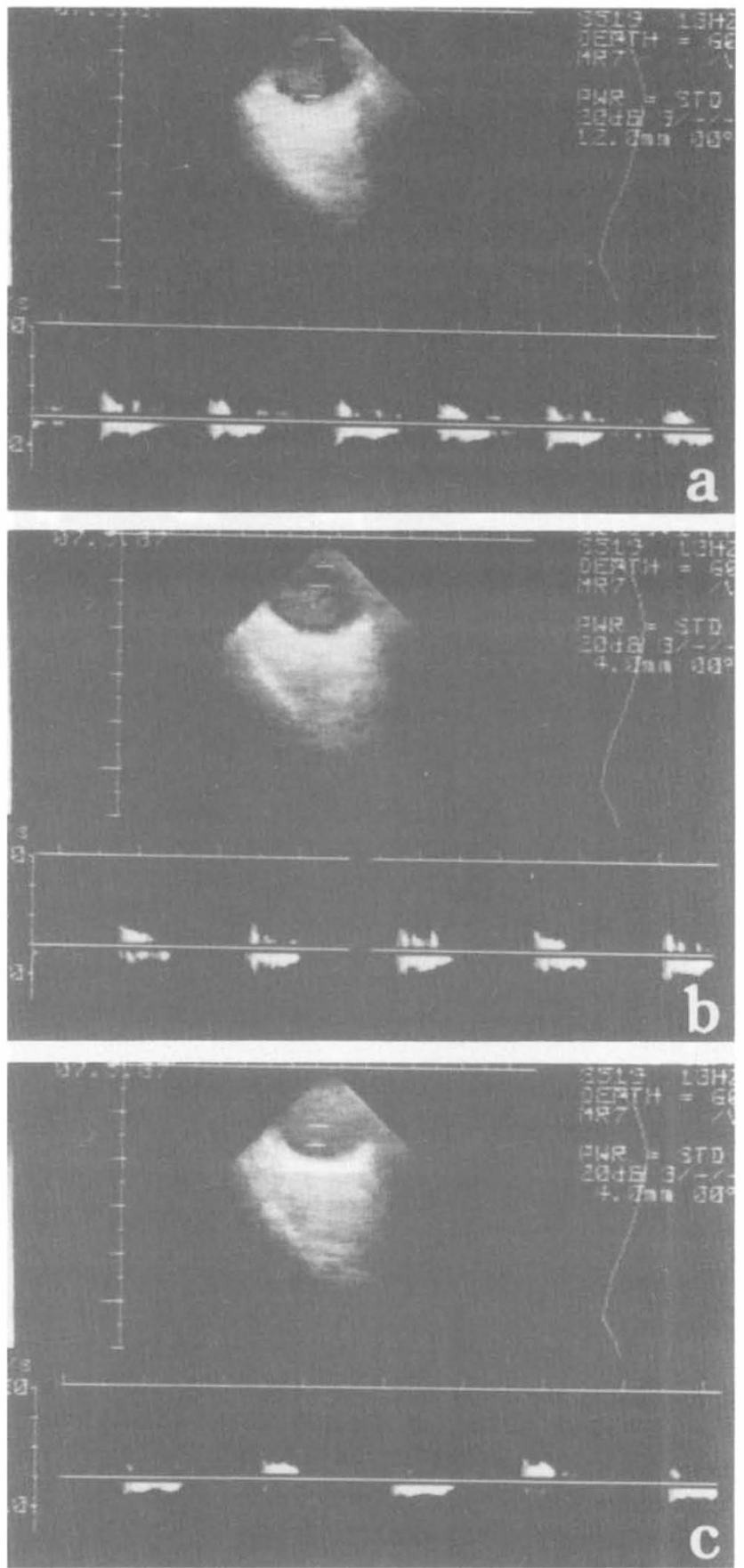

Fig. 2. Top: B-mode images from 'real-time' display. Two horizontal lines (gate) mark the region within which doppler studies are performed. Dashed line indicates direction of travel of ultrasonic pulses. Bottom: Corresponding doppler spectrograms for several alternate deviations of gaze to the left and right. (a) mobile detached gel (wide gate), (b) anterior gel (narrow gate), (c) central gel (narrow gate through rotational centre of eye). 
D. WONG AND M. RESTORI
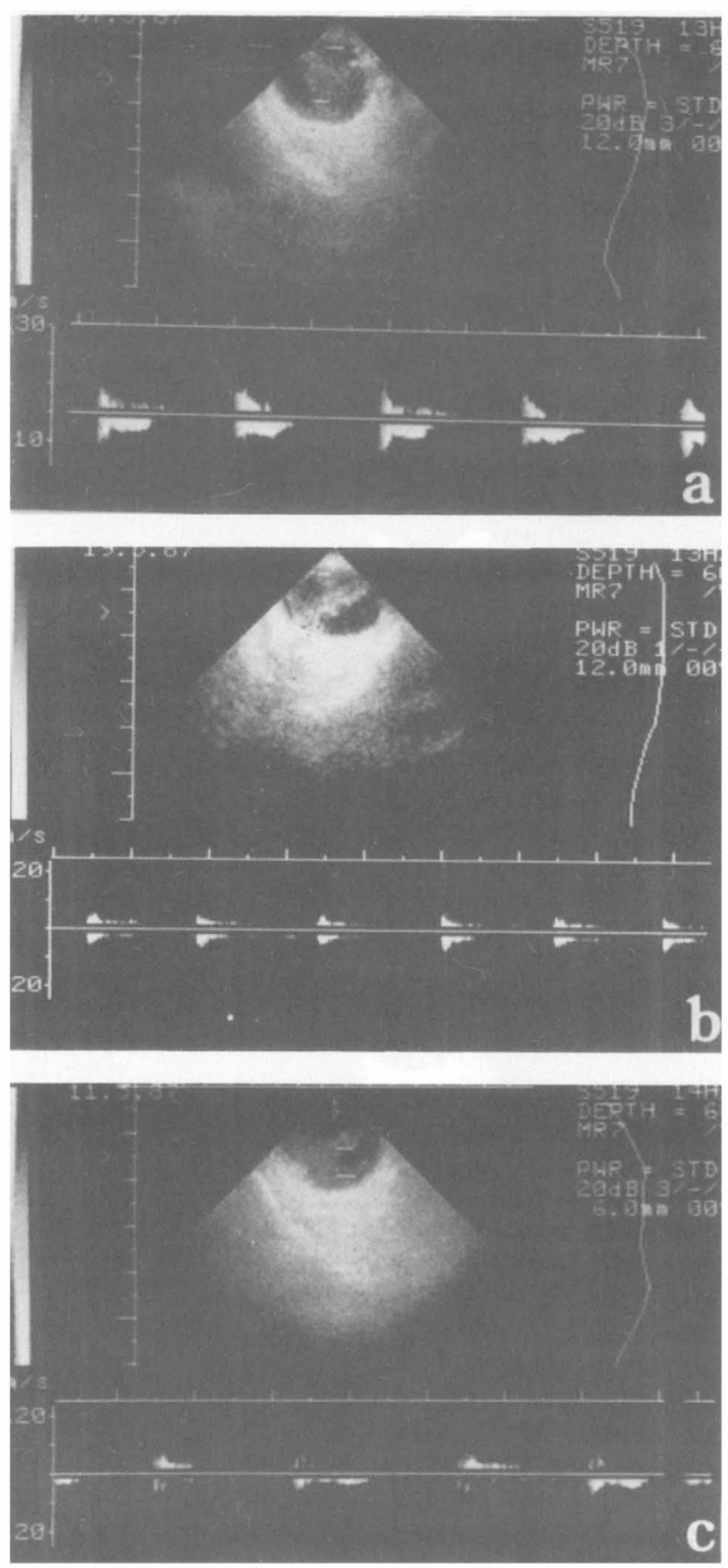

Fig. 3. B-mode images from 'real-time' display (gates mark regions of doppler study). Bottom: Corresponding spectrograms for several deviations of gaze to the left and right. (a) 'sloppy' gel. (b) 'firm' gel. (c) retrohyaloid opacity. 
came to a halt soon after the eye movement stopped. The difference between the 'stiff' and the 'sloppy' gel was marked and this difference was apparent both from the features of the spectrogram and from the characteristics of the audible signal.

\section{Retrohyaloid currents}

The retrohyaloid scatterers move with low velocities (Fig. 3c) and a characteristic swirling motion which generally persists longer than movements of detached vitreous gel.

\section{Discussion}

Machemer ${ }^{3}$ stated that rhegmatogenous retinal detachment resulted from a combination of the traction on the retina and of intraocular currents in the presence of a retinal hole. The dynamic vitreous tractional forces caused by eye movements cannot be directly measured. Using simultaneous 'real-time' B-mode and doppler techniques we have managed to record the velocities of scatterers within the vitreous gel and retrohyaloid space. It was found that the velocities of scatterers within a mobile vitreous gel were higher than those within the retrohyaloid space. In future, it might be possible to determine the acceleration and deceleration of scatterers using the same technique.

These preliminary studies have shown that it was possible to distinguish a 'firm' vitreous gel from a 'sloppy' one. The duration of motion of scatterers measured on the spectrogram trace was typically less than 0.5 secs for a 'stiff' gel and greater for a mobile gel; qualitatively, the features of the spectrogram and the audible doppler outputs associated with mobile and immobile gels were quite different. Measurement of accelerations or decelerations of gel scatterers may prove to be a better indicator of gel mobility than velocities of scatterers. Alternatively, the time taken for the maximum velocity to fall to half its value, may prove a useful measurement.

Further studies include use of this technique for looking at movement of detached vitreous gel and detached retina in cases of proliferative vitreoretinopathy. Clinically, the mobility of the retina and the vitreous as observed by ophthalmoscopy and biomicroscopy have been used to assess the severity of proliferation, to plan surgical treatment or indeed to decide upon operability. ${ }^{4}$ However, in cases with media opacity obscuring fundal view, such direct examination is often impossible and 'real-time' B-mode has already proved to be a useful adjunct. It is hoped that doppler techniques will provide useful indices to quantify the mobility of the vitreous and retina in these complex cases of retinal detachments and add to the information obtained with 'real-time' B-mode ultrasound.

\section{References}

${ }^{1}$ McLeod, D and Restori, M. Rapid B-scanning in ophthalmology. Clinical Diagnostic Ultrasound. Eds E. Barnett and P. Morley. Chapter 6. Blackwell series, Edinburgh (1985). p111-120.

2 Till, $P$ and Lessel, MR. Doppler techniques. Handbook of Clinical Ultrasound. Eds. de Vlieger, $\mathbf{M}$, Holmes, JH, Kazner, E, et al. John Wiley \& Sons, New York, 1978: 905-17.

${ }^{3}$ Machemer, R. The importance of fluid absorption, traction, intraocular currents, and chorioretinal scars in the therapy of rhegmatogenous retinal detachment. XLI Edward Jackson Memorial Lecture. Am J Ophthalmol, 1984, 98: 681-93.

${ }^{4} \mathrm{Jaffe}$, NS. The vitreous in clinical ophthalmology. C. V. Mosby Co, St Louis. 1969: 189-90. 